International Journal of Theoretical and Applied Finance

Vol. 4, No. 1 (2001) 1-21

(C) World Scientific Publishing Company

\title{
LOCAL SCALE INVARIANCE AND CONTINGENT CLAIM PRICING
}

\author{
J. K. HOOGLAND* and C. D. D. NEUMANN ${ }^{\dagger}$ \\ Centrum voor Wiskun de en Informatica (CWI), \\ P.O. Box 94079, 1090 GB Amsterdam, The Netherlands
}

Received 6 October 1999

Accepted 3 August 2000

\begin{abstract}
Prices of tradables can only be expressed relative to one another at any instant of time. This fundamental fact should therefore also hold for contingent claims, i.e. tradable instruments, whose prices depend on the prices of other tradables. We show that this property induces a local scale invariance in the problem of pricing contingent claims. Due to this symmetry we do not require any martingale techniques to arrive at the price of a claim. If the tradables are driven by Brownian motion, we find, in a natural way, that this price satisfies a PDE. Both possess a manifest gauge invariance. A unique solution can only be given when we impose restrictions on the drifts and volatilities of the tradables, i.e. the underlying market structure. We give some examples of the application of this PDE to the pricing of claims. In the Black-Scholes world we show the equivalence of our formulation with the standard approach. It is stressed that the formulation in terms of tradables leads to a significant conceptual simplification of the pricing-problem.
\end{abstract}

Keywords: Contingent claim pricing, scale invariance, homogeneity, partial differential equation.

\section{Introduction}

The essence of trading is the exchange of goods. Every transaction sets a ratio between the value of the two goods. This means that there is no such thing as the absolute value of an object, it can only be defined relative to the value of another object. If we only have one good, we cannot assign a price to the good. We need at least two goods. Then after choosing one of these two goods, the other good can be assigned a price relative to the first one. If we have $n+1$ tradable goods we can choose any of these $n+1$ tradables to assign a price to the other ones. The tradable that is chosen to set the prices of the other tradable is often called a numeraire. In fact, we have even more freedom. We can choose any positive-definitive function of the tradables as a numeraire and express every tradable price in terms of it, e.g. money.

\footnotetext{
*E-mail: jiri@cwi.nl

†E-mail: neumann@cwi.nl
} 
Thus a price is always given in terms of some unit of measurement. It is a measure-stick which is used to relate different objects. As long as everything is expressed in terms of this one unit prices can be compared. Whether we scale the unit does not matter, prices will scale accordingly. This scale invariance is of great importance. Not only the prices of tradables which are used to set up the basic economy should scale with a change in numeraire, but any derived tradable like contingent claims, depending on other tradables, should act in the same way. This leads in a natural way to the constraint that the price of a claim as a function of the underlying tradables should be homogeneous ${ }^{a}$ of degree 1 . Otherwise the economy is not well posed. Note that this symmetry holds independently of the driving stochastic factors.

The original approach to the "fair" pricing of contingent claims [2], in a geometric Brownian motion setting, was based on the derivation of a PDE for the claim value. To derive this PDE Black and Scholes constructed a "risk-free" portfolio consisting of the claim and underlying stock, where "risk-free" indicates that it does not fluctuate w.r.t. a particular numeraire, money. As this portfolio was "riskfree" it had to grow by the "risk-free" interest rate and this allowed them to derive a PDE. Their result was augmented by Merton [14], who put forward the replicating portfolio argument using stocks and bonds. Black and Scholes' argument started out with a broken symmetry, as they did not work with the tradables stock and bond but with stock only. Hence they did not observe the fundamental homogeneity property which is built into the theory.

Although Merton already noticed the homogeneity property for the case of a simple European warrant, it was apparently not recognized that this property should be an intrinsic property of any economy in which tradables and derivatives on these tradables have prices relative to some numeraire. More recently, Jamshidian [12] discussed interest-rate models and showed that if a payoff is a homogeneous function of degree 1 in the tradables, it leads naturally to self-financing trading strategies for interest-rate contingent claims. But again it is not appreciated that the homogeneity is a fundamental property, which any economy should possess to be properly defined.

The more recent and popular approach to "fair" pricing of a contingent claim [8] is based on martingales. Again the goal is to show the existence of a self-financing trading strategy which replicates the payoff of the claim at the maturity of the contract. If the economy does not allow for arbitrage and is complete, this self-financing trading strategy gives a unique price for the claim at any time. A key ingredient to arrive at this result is the use of the martingale representation theorem. One starts with the definition of the stochastic dynamics of the underlying tradables under the real-world measure. A numeraire is chosen to discount all tradables. Next one has to perform a change of measure, such that tradables, discounted by a numeraire,

${ }^{\text {a A function }} f\left(x_{0}, \ldots, x_{n}\right)$ is called homogeneous of degree $r$ if $f\left(a x_{0}, \ldots, a x_{n}\right)=$ $a^{r} f\left(x_{0}, \ldots, x_{n}\right)$. Homogeneous functions of degree $r$ satisfy the following property (Euler): $\sum_{\mu=0}^{n} x_{\mu} \frac{\partial}{\partial x_{\mu}} f\left(x_{0}, \ldots, x_{n}\right)=r f\left(x_{0}, \ldots, x_{n}\right)$. 
become martingales under this new measure. When this change of measure exists, one has to show that the discounted payoff of the claim is a martingale under this new measure too. Then the martingale representation theorem is invoked to link the discounted payoff martingale to the underlying discounted tradables. This then gives a self-financing trading strategy using the underlying (discounted) tradables, which replicates the (discounted) claim at all times and thus yields a (discounted) price for the claim. Finally one re-expresses everything in terms of the original variables. Note that one first has to find a way to transform the (discounted) tradables into martingales under some (rather) artificial measure to apply the martingale representation theorem, while at the end one re-expresses everything back in terms of the original tradables.

The fair price should be invariant under the choice of numeraire and it can indeed be shown this property holds under changes of measure, which are associated with different numeraires. Geman et. al. [7] used this invariance to show that, depending on the pricing problem at hand, it is useful to select a numeraire, which most naturally fits the payoff of the claim. They claim that one of the virtues of the martingale approach is that the whole pricing procedure and the numeraire invariance property are very clear.

In this article we argue that symmetry arguments should be put at the basis of any pricing theory, irrespective of the driving stochastic factors. The typical formulation using martingales is, in fact, possible by virtue of the local scale invariance and not the other way around. In fact, our main claim is that it is much more convenient and more natural to work in terms of SDE's and PDE's than to work with martingales. The homogeneity property circumpasses the usual change of measure argument and allows one to arrive at the usual pricing formula, in a Black-Scholes setting, under any fixed measure directly, without breaking the underlying local scale invariance. To arrive at this result, we use the sole fact, which has not been appreciated enough in the literature, that the definition of a market, or better its symmetry, puts a strong constraint on the dynamics of the tradables.

In this paper we start our discussion with the scale invariance of a frictionless economy of tradables with prices expressed in some arbitrary numeraire. Our next step is to define the stochastic dynamics of the prices of tradables. For clarity we will restrict ourselves to the case where the driving stochastic factors are Brownian motions. Also we assume the economy to be complete. Itô then leads to a SDE for a claim price. If the claim price solves the Kolgomorov's backward PDE then together with the homogeneity property this leads automatically to a self-financing trading strategy replicating the claim price. If no-arbitrage constraints are imposed on the drifts and volatilities of the stochastic prices, this price is unique. The invariance under changes of numeraire becomes very transparent due to the homogeneity property. We do not have to apply changes of measure and this leads in our view to a conceptually more satisfying and transparent contingent claim pricing argument. Finally the scale invariance property should be satisfied also in economies which do have friction. The symmetry invokes constraints which may be useful in 
model-building, e.g. more general stochastic processes. We will discuss this in a forthcoming publication. Also a more rigorous exposition of these results will be presented in a future publication. In the present paper, we want to focus on the main ideas and defer the mathematical details to a later time. To the best of our knowledge this is the first time that the importance and consequences of local scale invariance for contingent-claim pricing have been outlined and discussed.

The outline of the article is as follows. In Sec. 2 we introduce some standard notions used to price contingent claims in an economy with stochastic tradables. In Sec. 2.1 we show that for an economy to be properly defined it is required to be scale invariant. The scale invariance restricts the contingent claim price: it should be a homogeneous function of the underlying tradables of degree 1. In Sec. 2.2 we introduce the dynamics of the prices of tradables and introduce the notion of deterministic constraints on the dynamics, which may follow from certain choices for the drifts and volatilities of the tradables. We assume the stochastic driving factors to be Brownian motions in this article. In Sec. 2.3 we use the homogeneity together with Itô to derive the Kolgomorov's backward PDE for the contingent claim value. The homogeneity automatically insures the existence of a self-financing trading strategy for the contingent-claim in a Brownian motion setting. In Sec. 2.4 we show that the claim price will be unique if the constraints on the dynamics can be written as self-financing portfolios. Finally in Sec. 2.5 it is shown that the symmetry is inherited by the PDE for the claim value. This allows us to pick an appropriate numeraire (fix a gauge) and solve the PDE. Section 3 gives various applications of the PDE and the scale invariance in pricing of contingent claims. For more complex examples, such as path-dependent contigent claims we refer to Refs. [10] and [11]. In Sec. 3.1 we give the explicit formula for a European claim with log-normal prices for the underlying tradables. In Sec. 3.2 it is shown that the Black-Scholes PDE is contained in our approach. In Sec. 3.3 the pricing of quantos is discussed. In our formulation the pricing becomes trivial. In Sec. 3.4 we show that term-structure models fit naturally into our approach and give as an example the price of a log-normal stock in a Gaussian HJM model. Another example of the simple formulae is given in Sec. 3.5, where we consider a trigger-swap. Finally we give our conclusions and outlook in Sec. 4.

\section{Contingent Claim Pricing}

In the following subsections we will discuss some general properties of contingent claim pricing using dimensional analysis.

First let us recall the basic principles. We consider a frictionless market with $n+1$ tradables $^{\mathrm{b}}$ with prices $x_{\mu}$, where w $^{\mathrm{c}}=0, \ldots, n$. The prices $x \equiv\left\{x_{\mu}\right\}_{\mu=0}^{n}$ follow

b'Tradables are objects which are trivially self-financing: it neither costs nor yields money to keep a fixed amount of them. Examples are stocks and bonds. Note that money is not a tradable, unless the interest rate is zero.

cWe will always use Greek symbols $\mu, \nu, \kappa, \ldots$ for indices running from 0 to $n$ and Latin symbols $i, j, k, \ldots$ for indices running from 1 to $n$. 
stochastic processes, driven by Brownian motions ${ }^{\mathrm{d}}$. Time is continuous. Transaction costs are zero. Dividends are zero. Short positions in tradables are allowed. We want to value a European claim at time $t$ promising a payoff $f(x)$ at maturity $T>t$. To attach a rational price to the claim at time $t$ we have to find a dynamic portfolio or trading strategy $\phi \equiv\left\{\phi_{\mu}(x, t)\right\}_{\mu=0}^{n}$ of underlying tradables $x$ with value ${ }^{\mathrm{e}}$

$$
V(x, t)=\phi_{\mu}(x, t) x_{\mu},
$$

which replicates the payoff of the claim at maturity, $V(x, T)=f(x)$. Furthermore the trading-strategy has to be self-financing, i.e. we set up a portfolio for a certain amount of money today such that no further external cash-flows are required during the life-time of the contract to finance the payoff of the claim at maturity. All changes in the positions $\phi_{\mu}(x, t)$ at any given instant are financed by exchanging part of the tradables at current market prices for others such that the total cost is null. The self-financing property of the trading-strategy is expressed by:

$$
d V=\phi_{\mu} d x_{\mu} .
$$

If we can find such a trading-strategy, then the rational value of the claim today equals the value of the trading portfolio today. Finally we also have to impose the following restriction on the allowed trading strategies $\phi$ to be admissible: the value of a self-financing replicating portfolio is either deterministically zero at any time during the life of the contract or never. Otherwise arbitrage is possible. Uniqueness of the claim value only follows in special cases, i.e. for specific choices of stochastic dynamics and drifts and volatilities. We come back to this point in Sec. 2.4.

\subsection{Homogeneity}

For a market to exist we need at least two tradables. The price of one tradable is defined relative to the other tradable. It is however convenient to express their relative prices in terms of a numeraire. The numeraire may be any positive-definite, possibly stochastic, function. The freedom to choose an arbitrary numeraire implies the existence of a local scale symmetry for prices of tradables. The symmetry automatically implies the existence of a delta-hedging strategy for any tradable which depends on other underlying tradables.

Let us consider again a market with $n+1$ basic tradables with prices $x$ at time $t$. These prices are in units $U$ of the numeraire. We say that the $x$ have dimension $U$, or symbolically $\left[x_{\mu}\right]=U$. For the moment we leave the dynamics unspecified. What can be said about the price of a claim today, again in units of $U$, when expressed in terms of the tradables $x$ ? Let us denote the price of the claim by $V(x, t)$. Just

\footnotetext{
${ }^{\mathrm{d}}$ More general processes will be discussed in a future publication.

'We use Einstein's summation convention: repeated indices in products are summed over unless stated otherwise.
} 
on the basis of dimensional analysis we can write down the following form for the price

$$
V(x, t)=\phi_{\mu}(x, t) x_{\mu} .
$$

Since $[V]=U$ and $\left[x_{\mu}\right]=U$, the functions $\phi_{\mu}$ are dimensionless, $\left[\phi_{\mu}\right]=1$. This implies that they can only be functions of ratios of different tradables, which are again dimensionless.

The same arguments apply to any payoff function, for otherwise it is ill-specified. For example, the payoff function of a vanilla call with maturity $T$ does not seem to have this form at first sight

$$
(S(T)-K)^{+}
$$

But what is meant is the following function of a stock $S(t)$ and a discount bond $P(t, T)$, which pays 1 unit of $U$ at time $T$

$$
(S(T)-K P(T, T))^{+},
$$

and this does have the right form.

Now suppose that we change our unit of measurement. If we scale the unit by $a$, such that $U \rightarrow U / a$, then the prices of the tradables will scale accordingly, $x_{\mu} \rightarrow a x_{\mu}$. Using the dimensional analysis result above we then find the following property for the price of the claim

$$
V(a x, t)=\phi_{\mu}(a x, t) a x_{\mu}=a \phi_{\mu}(x, t) x_{\mu}=a V(x, t) .
$$

The price of the claim is a homogeneous function of degree 1 . Note that the scale factor $a$ may be local, $a=a(x, t)$. Differentiating Eq. (2.6) with respect to $a$, this immediately yields the following relation, valid for any homogeneous function ${ }^{\mathrm{f}}$ of degree 1 ,

$$
V(x, t)=x_{\mu} \frac{\partial V(x, t)}{\partial x_{\mu}} \equiv x_{\mu} \partial_{x_{\mu}} V(x, t) .
$$

This result is independent of the choice of dynamics. Even if we relax the frictionless market assumptions, this local scale invariance should not be broken.

As already mentioned various authors $[12,14]$ have already touched upon the homogeneity property of certain claim prices, but they always inferred this property as a consequence of the no-arbitrage conditions they imposed on the drift and volatilities of the tradables. Furthermore their claim is that this property only holds in certain cases. In fact Jamshidian [12] gives a theorem which is very similar to what we discuss in Sec. 2.3, except that he doesn't recognize the fact that the required homogeneity should always be satisfied. This should be contrasted with our presentation above, where we show that this homogeneity property is one of the most fundamental properties any market model must posses to be well-posed. The homogeneity property just expresses the fact that one needs a proper coordinatesystem. It could be termed: "the relativity principle of finance".

\footnotetext{
${ }^{\mathrm{f}}$ We allow generalized functions.
} 


\subsection{Dynamics: The market model}

The prices of tradables, relative to a numeraire, change over time. Let us assume that the dynamics of the tradables is given by the following stochastic differential equation:

$$
d x_{\mu}(t)=x_{\mu}(t)\left(\sigma_{\mu}(x, t) \cdot d W(t)+\alpha_{\mu}(x, t) d t\right), \quad \text { (no sum) }
$$

where we have $k$ independent standard Brownian motions driving the $n$ tradables and initial conditions ${ }^{\mathrm{g}} x_{\mu}(t)$. Note here no summation over $\mu$ is assumed. The Brownian motion is defined under some measure, associated with some arbitrary numeraire. For the pricing argument the choice of numeraire is completely irrelevant. To determine a price for the claim we will always work under one measure, while keeping the numeraire invariance manifest. This should be contrasted with the usual approach, where one first applies a change of measure to make the tradables martingales under the new measure. Then one invokes the martingale representation theorem to determine the claim price. This change of measure is not required, as we will show later, for the determination of a rational price. In fact we do not even have to require the tradables to be strictly positive. If one of the tradables would become zero, this is allowed as long as it hits zero in a non-deterministic way. The tradable should not be used as a numeraire.

For the properties of the drift and volatilities we refer to the appendix. Both the LHS and RHS have dimension $U$. It is convenient to extract a unit of $x_{\mu}$ from the drift and volatilities in Eq. (2.8) to make them dimensionless ${ }^{\text {h }}$ Thus the only allowed form for the drift and volatility-structure are functions of the ratios of the tradables. This is a fundamental requirement for any viable and properly posed market model.

At first sight, one might think that this restriction on the drift and volatility terms excludes some well-known models for the prices of tradables, such as ordinary Brownian motion or mean-reverting (Ornstein-Uhlenbeck) models. This, however, is not true. To make this fact clear, let us consider a simple example, a market in which there are only two tradables, call them $S$ and $P$. If we take $P$ as numeraire, then its price obviously satisfies

$$
d P(t)=0
$$

This expresses the fact that $P$ has a constant value when expressed in terms of itself. Given this numeraire, we can consider a number of models for the price of $S$. For example,

$$
d S(t)=S(t)(\lambda d t+\sigma d W(t))
$$

g Here $\sigma_{\mu}$ and $d W$ should be understood as $k$-dimensional vectors. We denote the inner product by a dot.

${ }^{\mathrm{h}}$ In the literature the $\alpha_{\mu}$ and $\sigma_{\mu}$ are often called relative drift and volatilities. 
leads to geometric Brownian motion, while

$$
d S(t)=P(t)(\lambda d t+\sigma d W(t))
$$

gives rise to ordinary Brownian motion. And an Ornstein-Uhlenbeck process can be described by

$$
d S(t)=(\lambda P(t)-\kappa S(t)) d t+\sigma P(t) d W(t) .
$$

In all cases, $\lambda, \kappa$ and $\sigma$ are dimensionless constants. Obviously, all these models can be written in the form of Eq. (2.8) So this does not lead to a restriction on the market model. Rather it forces one to think about the numeraire dependencies of these models.

A priori it could well be that deterministic relations exist between the tradables. These relations should satisfy certain constraints in order to attach a unique rational price to a claim. If these constraints are satisfied, arbitrage is not possible. We will come back to this point in Sec. 2.4.

\subsection{Deriving the basic $P D E$}

The results of the previous sections are precisely what is needed to obtain the Kolgomorov's backward PDE for the price of a contingent claim. It will be shown that the homogeneity property, together with this PDE, is all that is necessary to obtain a unique self-financing trading-strategy in an arbitrage-free market. We do not have to make a detour using martingale techniques to prove this fact. This is a substantial conceptual simplification of the standard theory.

Let us consider the evolution of the contingent claim price $V(x, t)$ in time. Using Itô we arrive at the following SDE

$$
d V=\partial_{x_{\mu}} V d x_{\mu}+\left(\partial_{t}+\frac{1}{2} \sigma_{\mu} \cdot \sigma_{\nu} x_{\mu} x_{\nu} \partial_{x_{\mu}} \partial_{x_{\nu}}\right) V d t
$$

At this point the homogeneity property of $V(x, t)$ is used. Since

$$
V=x_{\mu} \partial_{x_{\mu}} V,
$$

we see that if the claim value solves the PDE

$$
\mathcal{L} V \equiv\left(\partial_{t}+\frac{1}{2} \sigma_{\mu} \cdot \sigma_{\nu} x_{\mu} x_{\nu} \partial_{x_{\mu}} \partial_{x_{\nu}}\right) V=0
$$

a replicating portfolio, containing $\partial_{x_{\mu}} V$ of tradable $x_{\mu}$, is indeed self-financing.

$$
d V=\partial_{x_{\mu}} V d x_{\mu}
$$

As usual, the payoff of the claim is specified as the boundary condition of the PDE.

Note that the drift terms did not enter the derivation of the PDE at all. We did not have to apply a change of measure to obtain an equivalent martingale measure and use the martingale representation theorem. All that was needed was the homogeneity of the contingent claim price as a function of the underlying tradables. 
The PDE in Eq. (2.15) provides, in our view, the most natural formulation of the valuation of claims on tradables in a Brownian motion setting. It allows us to easily derive the classical result of Black, Scholes, and Merton (Sec. 3.2), but also the results of Heath-Jarrow-Morton (Sec. 3.4). Although we considered European claims up till now, it is not too difficult to include path-dependent properties. This is discussed in Refs. [10] and [11].

\subsection{Uniqueness: No arbitrage revisited}

In the previous subsection we showed that if the claim value solves Eq. (2.15) then the replicating portfolio for the claim is self-financing. If deterministic relations between tradables exist, this is too strong a condition. In that case the constraints introduce a redundancy (gauge-freedom) in the space of tradables. This implies that we only have to solve $\mathcal{L} V=0$ modulo the constraints. The deterministic relations between tradables allow the construction of deterministic portfolios with zero value for all times. We will call them null-portfolios. Suppose that there exist $m$ deterministic relations

$$
P_{i}(t)=\psi_{i, \mu}(x, t) x_{\mu}=0,
$$

with $i=1, \ldots, m$. We will assume for the moment that these relations are independent such that they span the null-space $\mathcal{P}$. Otherwise we can find a smaller set of independent constraints to span the null-space. We also assume that the dimension of the null-space is constant over time. Thus we can write the null-space $\mathcal{P}$ as follows:

$$
\mathcal{P}=\left\{f_{i}(x, t) P_{i}(t) \mid \operatorname{arbitrary} f_{i}(x, t)\right\},
$$

where the $f_{i}$ are predictable homogeneous functions of degree 0 of the prices. Taking into account the constraints we require

$$
\mathcal{L} V \approx 0 .
$$

Here we use the notation $\approx 0$ to write $\mathcal{L} V=0$ modulo elements in the null-space $\mathcal{P}$.

The null-portfolios are either self-financing or not. In the first case, the price of the claim is unique up to arbitrary null-portfolios for all times. No external cashflows are required to keep the null-portfolio null. In the second case we can find two portfolios which replicate the payoff at maturity but whose values diverge as one moves away from maturity. There will be no unique price and arbitrage is possible.

A market will have self-financing null-portfolios if the drift and volatilities satisfy certain constraints. A null-portfolio $P=\psi_{\mu} x_{\mu} \in \mathcal{P}$ satisfies by definition

$$
d P \approx 0 \text {. }
$$

Since the null-portfolio is by definition deterministic, this leads automatically to the following constraints on the volatilities

$$
\frac{\partial P}{\partial x_{\mu}} \sigma_{\mu} x_{\mu}=\psi_{\mu} \sigma_{\mu} x_{\mu}+\frac{\partial \psi_{\nu}}{\partial x_{\mu}} \sigma_{\mu} x_{\mu} x_{\nu} \approx 0 .
$$


If a null-portfolio is self-financing, we have

$$
d P=\psi_{\mu} d x_{\mu} .
$$

But Eq. (2.20) immediately gives

$$
\psi_{\mu} d x_{\mu} \approx 0
$$

which implies

$$
\begin{aligned}
& \psi_{\mu} \alpha_{\mu} x_{\mu} \approx 0, \\
& \psi_{\mu} \sigma_{\mu} x_{\mu} \approx 0 .
\end{aligned}
$$

If these constraints are satisfied for all null-portfolios, then the null-portfolios will be self-financing and hence no arbitrage is possible.

As a simple example of such constraints, let us consider two tradables $x_{1,2}$ with one Brownian motion

$$
\frac{d x_{1,2}}{x_{1,2}}=\sigma_{1,2} d W(t)+\alpha_{1,2} d t
$$

and constant drifts $\alpha_{1,2}$ and volatilities $\sigma_{1,2}$ and initial values $x_{1,2}(0)=1$. Note that this is the usual setting of Black-Scholes. The SDE for the ratio $x_{2} / x_{1}$ then becomes

$$
\frac{d x_{2} / x_{1}}{x_{2} / x_{1}}=\left(\sigma_{2}-\sigma_{1}\right) d W+\left(a_{2}-\alpha_{1}-\sigma_{1}\left(\sigma_{2}-\sigma_{1}\right)\right) d t .
$$

If the tradables satisfy a deterministic relation, we see that this is only possible if the volatilities are equal, $\sigma_{1}=\sigma_{2} \equiv \sigma$. In that case the above SDE reduces to an ODE

$$
\frac{d x_{2} / x_{1}}{x_{2} / x_{1}}=\left(\alpha_{2}-\alpha_{1}\right) d t
$$

Solving the ODE, we find the following deterministic relation

$$
x_{2}(t)=x_{1}(t) e^{\left(\alpha_{2}-\alpha_{1}\right) t} .
$$

The existence of this relation allows us to construct a null-portfolio with zero value and previsible coefficients for all times. Indeed

$$
P(t)=x_{2}(t)-x_{1}(t) e^{\left(\alpha_{2}-\alpha_{1}\right) t}
$$

is trivially zero. Two cases can be distinguished. The portfolio $P$ is self-financing or it is not. Consider the evolution of $P$

$$
d P=d x_{1}-e^{\left(\alpha_{2}-\alpha_{1}\right) t} d x_{2}+\left(\alpha_{2}-\alpha_{1}\right) e^{\left(\alpha_{2}-\alpha_{1}\right) t} x_{1} d t .
$$

It should be clear that only if $\alpha_{1}=\alpha_{2}$ will the portfolio $P$ be self-financing and can $x_{1}$ be hedged using $x_{2}$. Otherwise arbitrage is possible. Intuitively this should be obvious, two tradables with equal risk $\sigma$ should yield the same return $\alpha$. 
Let us consider the consequences for the price $V$ of a claim if $\alpha_{1} \neq \alpha_{2}$. We construct a portfolio with constant coefficients $\psi_{1,2}$ and price process

$$
P(t)=\psi_{1} x_{1}(t)+\psi_{2} x_{2}(t) .
$$

If we set

$$
\psi_{2}=-\psi_{1} e^{\left(a_{1}-\alpha_{2}\right) T},
$$

then the value of the portfolio at time $T$ is $P(T)=0$. However at $t<T$ we have

$$
P(t)=\psi_{1} x_{1}(t)\left(1-e^{\left(a_{1}-\alpha_{2}\right)(T-t)}\right) .
$$

Since $\psi_{1}$ can take any value, the value of the contract which pays zero at time $T$ can have any value. But this implies that we can ask any price $V(t)+P(t)$ for a claim paying $V(T)$ by adding an arbitrary portfolio with $P(T)=0$.

\subsection{Gauge invariance of the PDE}

It was shown that a fundamental property of any viable market-model is the local scale invariance of the prices of tradables as expressed through the freedom of choice of the numeraire. It leads automatically to the requirement that the claim price should be a homogeneous function of degree 1 in terms of prices of tradables.

The scale invariance of the claim price is inherited by the PDE via an invariance of the solutions of the PDE under a simultaneous shift of all volatility functions by an arbitrary function $\lambda(x, t)$

$$
\sigma_{\mu}(x, t) \rightarrow \sigma_{\mu}(x, t)-\lambda(x, t) .
$$

Indeed, if $V$ solves $\mathcal{L} V=0$, then it also solves

$$
\left(\partial_{t}+\frac{1}{2}\left(\sigma_{\mu}(x, t)-\lambda(x, t)\right) \cdot\left(\sigma_{\nu}(x, t)-\lambda(x, t)\right) x_{\mu} x_{\nu} \partial_{x_{\mu}} \partial_{x_{\nu}}\right) V=0 .
$$

This can easily be checked by noting that for homogeneous functions of degree 1 we have

$$
x_{\mu} \partial_{x_{\mu}} \partial_{x_{\nu}} V=0 .
$$

This ensures that terms involving $\lambda$ drop out of the PDE. (Note that this equation gives interesting relations between the various $\Gamma$ 's of the claim). From this it follows that $V$ itself must be invariant under the substitution defined by Eq. (2.35). This corresponds to the freedom of choice of a numeraire. It just states that volatility is a relative concept. Price functions should not depend on the choice of a numeraire.

We can exploit this freedom to reduce the dimension of the problem. For example, choosing $x_{0}$ as a numeraire corresponds to taking $\lambda(x, t)=\sigma_{0}(x, t)$. Then

$$
\left(\partial_{t}+\frac{1}{2}\left(\sigma_{i}(x, t)-\sigma_{0}(x, t)\right) \cdot\left(\sigma_{j}(x, t)-\sigma_{0}(x, t)\right) x_{i} x_{j} \partial_{x_{i}} \partial_{x_{j}}\right) V=0
$$


Now one can introduce

$$
V\left(x_{0}, \ldots, x_{n}, t\right)=x_{0} E\left(\frac{x_{1}}{x_{0}}, \ldots, \frac{x_{n}}{x_{0}}, t\right) .
$$

Then $E\left(x_{1}, \ldots, x_{n}, t\right)$ again satisfies Eq. (2.38). Interesting things happen when $V$ is independent of $x_{0}$. In that case, $E$ is homogeneous again, the $\sigma_{0}(x, t)$ dependence drops out, and the game can be repeated. Furthermore it should be noted, that the numeraire does not have to be a tradable. As stated earlier it may be be any positive-definite stochastic function. This freedom can be exploited to simplify calculations.

\section{Applications}

In this section we give several examples which show the simplicity and clarity with which one derives results for contingent claim prices using the scale invariance of the PDE.

\subsection{General solution for the log-normal case}

We compute the claim price for a path-independent European claim with an arbitrary number of underlying tradables, when the prices of the tradables are lognormally distributed,

$$
\frac{d x_{\mu}}{x_{\mu}}=\sigma_{\mu}(t) \cdot d W(t)+\alpha_{\mu}(t) d t, \quad(\text { no sum })
$$

It is easy to write the general solution for a path-independent European claim in this case. First we perform a change of variables

$$
x_{\mu}=\exp \left(y_{\mu}\right) \text {, }
$$

such that the PDE becomes

$$
\left(\partial_{t}+\frac{1}{2} \sigma_{\mu}(t) \cdot \sigma_{\nu}(t)\left(\partial_{y_{\mu}} \partial_{y_{\nu}}-\delta_{\mu \nu} \partial_{y_{\mu}}\right)\right) V=0 .
$$

A Fourier transformation yields an ODE in $t$

$$
\left(\partial_{t}-\frac{1}{2} \sigma_{\mu}(t) \cdot \sigma_{\nu}(t)\left(\tilde{y}_{\mu} \tilde{y}_{\nu}-i \delta_{\mu \nu} \tilde{y}_{\mu}\right)\right) \tilde{V}=0,
$$

where $i$ denotes the imaginary unit. The ODE has the solution

$$
\tilde{V}(t)=\tilde{V}(T) \exp \left(-\frac{1}{2} \Sigma_{\mu \nu}\left(\tilde{y}_{\mu} \tilde{y}_{\nu}-i \delta_{\mu \nu} \tilde{y}_{\mu}\right)\right),
$$

with

$$
\Sigma_{\mu \nu} \equiv \int_{t}^{T} \sigma_{\mu}(u) \cdot \sigma_{\nu}(u) d u
$$


Clearly $\Sigma$ is a non-negative symmetric matrix. If we perform a singular value decomposition, we get

$$
\Sigma_{\mu \nu}=A_{\mu \sigma} A_{\nu \rho} B_{\sigma \rho}, \quad B=\operatorname{diag}\left(\lambda_{0}, \ldots, \lambda_{m-1}, 0, \ldots\right),
$$

where $A$ is an orthogonal matrix and $m$ equals the rank of $\Sigma$ (so $\lambda_{i}>0$ for $0 \leq i<m)$. It will turn out to be convenient to introduce the matrix

$$
\Theta_{\mu \nu}=\left\{\begin{array}{ll}
A_{\mu \nu} \sqrt{\lambda_{\nu}} & \text { for } \nu<m \\
A_{\mu \nu} & \text { otherwise }
\end{array} .\right.
$$

Clearly, this matrix is invertible, $\operatorname{det} \Theta=\sqrt{\lambda_{0} \cdots \lambda_{m-1}}$, and it satisfies

$$
\Sigma_{\mu \nu}=\Theta_{\mu \sigma} \Theta_{\nu \rho} \Lambda_{\sigma \rho}, \quad \Lambda=\operatorname{diag}(\underbrace{1, \ldots, 1}_{m}, 0, \ldots) .
$$

We now perform an inverse Fourier transformation on the solution of the ODE, and find

$$
\begin{aligned}
V\left(x_{0}, \ldots, x_{n}, t\right)= & \frac{1}{(2 \pi)^{n+1}} \iint V\left(\exp \left(y_{0}\right), \ldots, T\right) \\
& \times \exp \left(-\frac{1}{2} \Sigma_{\mu \nu}\left(\tilde{y}_{\mu} \tilde{y}_{\nu}-i \delta_{\mu \nu} \tilde{y}_{\mu}\right)+i \tilde{y}_{\mu}\left(y_{\mu}-\ln x_{\mu}\right)\right) d y d \tilde{y} \\
= & \frac{1}{(2 \pi)^{n+1}} \iint V\left(x_{0} \exp \left(y_{0}-\frac{1}{2} \Sigma_{00}\right), \ldots, T\right) \\
& \times \exp \left(-\frac{1}{2} \Sigma_{\mu \nu} \tilde{y}_{\mu} \tilde{y}_{\nu}+i \tilde{y}_{\mu} y_{\mu}\right) d y d \tilde{y} .
\end{aligned}
$$

Next we introduce new variables as follows

$$
y_{\mu}=\Theta_{\mu \nu} z_{\nu}, \quad \Theta_{\mu \nu} \tilde{y}_{\mu}=\tilde{z}_{\nu} .
$$

In terms of these variables, the integral becomes (note that the Jacobian of this transformation exactly equals one)

$$
\begin{aligned}
& \frac{1}{(2 \pi)^{n+1}} \iint V\left(x_{0} \exp \left(\Theta_{0 \nu} z_{\nu}-\frac{1}{2} \Sigma_{00}\right), \ldots, T\right) \\
& \times \exp \left(-\frac{1}{2} \Lambda_{\mu \nu} \tilde{z}_{\mu} \tilde{z}_{\nu}+i \tilde{z}_{\mu} z_{\mu}\right) d z d \tilde{z}
\end{aligned}
$$

The integral over the $\tilde{z}_{\mu}$ can be calculated explicitly. It gives rise to an $m$ dimensional standard normal PDF, multiplied by some $\delta$-functions

$$
\begin{gathered}
\frac{1}{(2 \pi)^{n+1}} \int \exp \left(-\frac{1}{2} \Lambda_{\mu \nu} \tilde{z}_{\mu} \tilde{z}_{\nu}+i \tilde{z}_{\mu} z_{\mu}\right) d \tilde{z}=\phi(z) \delta\left(z_{m}\right) \cdots \delta\left(z_{n}\right) \\
\phi(z)=\frac{1}{(\sqrt{2 \pi})^{m}} \exp \left(-\frac{1}{2} \sum_{i=0}^{m-1} z_{i}^{2}\right) .
\end{gathered}
$$


The integrals over $z_{\mu}$ for $\mu \geq m$ are now trivial. To express the result in a compact form, it is useful to introduce a set of $m$-dimensional vectors

$$
\left(\theta_{\mu}\right)_{i}=\Theta_{\mu i}, \quad 0 \leq i<m .
$$

These vectors in fact define a Cholesky-decomposition of the covariance matrix. Indeed, they satisfy

$$
\theta_{\mu} \cdot \theta_{\nu}=\Sigma_{\mu \nu}
$$

Here the inner product is understood to be $m$-dimensional. Combining all, the solution becomes

$$
V\left(x_{0}, \ldots, x_{n}, t\right)=\int \phi(z) V\left(x_{0} \exp \left(\theta_{0} \cdot z-\frac{1}{2} \theta_{0} \cdot \theta_{0}\right), \ldots, T\right) d^{m} z
$$

Since $V$ is homogeneous, the result can be expressed in an even more compact form

$$
V\left(x_{0}, \ldots, x_{n}, t\right)=\int V\left(x_{0} \phi\left(z-\theta_{0}\right), \ldots, x_{n} \phi\left(z-\theta_{n}\right), T\right) d^{m} z .
$$

If the number of tradables is small we may be able to compute Eq. (3.17) analytically. Otherwise we have to use numerical techniques.

At this point let us remind the reader that it is easy to include stocks in the model with known future dividend yields. This can be done as follows. Suppose we want to price a European claim $V$, whose price depends on a dividend paying stock $S$. The dividend payments occur at times $t_{i}, 1 \leq i \leq n$ during the lifetime of the claim. These dividends are given as a fraction $\delta_{i}$ of the stock-price $S\left(t_{i}\right)$. The effect of the dividend payments on the price of the claim can be incorporated by making the substitution

$$
S(t) \rightarrow S(t) \prod_{i=1}^{n}\left(1+\delta_{i}\right)^{-1}
$$

in the price function of a similar claim, but depending on a non-dividend paying stock. Indeed, a dividend payment at time $t_{i}$ has the effect of reducing the stock-price by a factor $\left(1+\delta_{i}\right)^{-1}$. For dividends paid at a continuous rate $q$, the substitution simply becomes

$$
S(t) \rightarrow S(t) e^{-q(T-t)}
$$

If dividend payments are known in terms of another tradable, e.g. a bond, the situation becomes more complicated. This is so because a dividend payment of $\delta_{i}$ units of a tradable $P$ at time $t_{i}$ has the effect of reducing the stock-price by a factor

$$
\left(1+\delta_{i} \frac{P\left(t_{i}\right)}{S\left(t_{i}\right)}\right)^{-1} \text {. }
$$

This makes the correction factor on $S$ path-dependent in general. We return to this problem in Ref. [11]. 


\subsection{Recovering Black-Scholes}

In Sec. 2.3 we derived a very general PDE for the pricing of contingent claims, when the stochastic terms are driven by Brownian motion. In this section we show that it reduces to the standard Black-Scholes equation when the underlying tradables are log-normally distributed with constant drift and volatilities. In the Black-Scholes world, we have a number of stocks $S_{i}$ with SDE's

$$
\frac{d S_{i}}{S_{i}}=\sigma_{i} \cdot d W(t)+\alpha_{i} d t .
$$

Furthermore we have a deterministic bond $P$, satisfying

$$
\frac{d P(t, T)}{P(t, T)}=r d t
$$

with $P(T, T)=1$. For simplicity we take the interest rate and volatilities to be time-independent. It is not too difficult to extend the present discussion to the time-dependent case. In fact the solution was already computed in the previous section. Our basic equation, Eq. (2.15), gives for the price of a claim

$$
\left(\partial_{t}+\frac{1}{2} \sigma_{i} \cdot \sigma_{j} S_{i} S_{j} \partial_{S_{i}} \partial_{S_{j}}\right) V=0
$$

Note that $V$ is explicitly a function of $P$. In the Black-Scholes formulation it is usually defined implicitly. This can be done by defining

$$
\begin{aligned}
E(S, t) & =V(P, S, t) \\
V(1, S, t) & =\frac{E(P(t) S, t)}{P(t)} .
\end{aligned}
$$

Thus we find, as promised,

$$
\left(\partial_{t}+r S_{i} \partial_{S_{i}}+\frac{1}{2} \sigma_{i} \cdot \sigma_{j} S_{i} S_{j} \partial_{S_{i}} \partial_{S_{j}}-r\right) E=0
$$

Let us now consider a simple one-dimensional example - a European call option. The solution can be easily found using the results of the previous section.

$$
V(P, S, t)=\int(S \phi(z-\sigma \sqrt{T-t})-K P \phi(z))^{+} d z=S \Phi\left(d_{1}\right)-K P \Phi\left(d_{2}\right),
$$

with

$$
d_{1,2}=\frac{\log \frac{S}{K P}}{\sigma \sqrt{T-t}} \pm \frac{1}{2} \sigma \sqrt{T-t} .
$$

This is the well-known Merton's formula [7]. The homogeneity relation, Eq. (2.7), can be used to derive relations between the greeks. In this case it is given by

$$
V=S \partial_{S} V+P \partial_{P} V
$$

Indeed, using $\partial_{S} V=\Phi\left(d_{1}\right)$ and $\partial_{P} V=-K \Phi\left(d_{2}\right)$, the equality follows. Since in the Black-Scholes universe $P$ is a deterministic function of $r$, we have for $\rho \equiv \partial_{r} V$

$$
\rho=\partial_{P} V \partial_{r} P=-(T-t) P \partial_{P} V=(T-t)\left(S \partial_{S} V-V\right)
$$


This type of relations were already observed in a different context in Ref. [4]. Furthermore, Eq. (2.37) gives the following relations

$$
S \partial_{S}^{2} V+P \partial_{P} \partial_{S} V=S \partial_{S} \partial_{P} V+P \partial_{P}^{2} V=0 .
$$

Again this is easily checked by substitution of the solution $V$ in Eq. (3.31).

\subsection{Quantos}

Quantos are instruments which have a payoff specified in one currency and payout in another currency. The pricing of these instruments becomes trivial, when we consider the problem using only tradables in one economy. This requires the introduction of an exchange rate to relate the instruments denominated in one currency to those denominated in another currency. The exchange rate is assumed to be stochastic and driven by Brownian motion. Let us denote the exchange rate to convert currency 2 into currency 1 by $C_{12}$, satisfying

$$
\frac{d C_{12}}{C_{12}}=\sigma_{12} \cdot d W(t)+\alpha_{12} d t
$$

The exchange rate $C_{21}=C_{12}^{-1}$ to convert currency 1 into currency 2 then satisfies

$$
\frac{d C_{21}}{C_{21}}=-\sigma_{12} \cdot d W(t)+\left(-\alpha_{12}+\sigma_{12}^{2}\right) d t .
$$

Let us consider two assets, one denominated in currency 1 and the other in currency 2 , with the following dynamics respectively $(i=1,2)$,

$$
\frac{d x_{i}}{x_{i}}=\sigma_{i} \cdot d W(t)+\alpha_{i} d t .
$$

To be able to price the instrument we need two tradables denominated in one currency. Let us define the converted prices $\tilde{x}_{1}=C_{21} x_{1}$ and $\tilde{x}_{2}=C_{12} x_{2}$. The converted prices give us our pairs of tradables $x_{1}, \tilde{x}_{2}$ and $\tilde{x}_{1}, x_{2}$ needed to price the instrument. The price is identical whether we work in terms of currency 1 or 2 . This is a direct consequence of the scale invariance of the problem. For consider first the case where everything is denoted in terms of currency 1 . Then we arrive at the following two SDE's

$$
\begin{aligned}
& \frac{d x_{1}}{x_{1}}=\sigma_{1} \cdot d W(t)+\alpha_{1} d t \\
& \frac{d \tilde{x}_{2}}{\tilde{x}_{2}}=\left(\sigma_{2}+\sigma_{12}\right) \cdot d W(t)+\left(\alpha_{2}+\alpha_{12}+\frac{1}{2} \sigma_{2} \sigma_{12}\right) d t .
\end{aligned}
$$

Thus the volatilities entering the pricing problem are $\sigma_{1}$ and $\tilde{\sigma}_{2} \equiv \sigma_{2}+\sigma_{12}$. Next, consider the case where we denominate everything in terms of currency 2 . The SDE's become

$$
\begin{aligned}
& \frac{d \tilde{x}_{1}}{\tilde{x}_{1}}=\left(\sigma_{1}-\sigma_{12}\right) \cdot d W(t)+\left(\alpha_{1}-\alpha_{12}+\sigma_{12}^{2}-\frac{1}{2} \sigma_{1} \sigma_{12}\right) d t \\
& \frac{d x_{2}}{x_{2}}=\sigma_{2} \cdot d W(t)+\alpha_{2} d t
\end{aligned}
$$


In this case, the volatilities which are relevant for the pricing problem are $\sigma_{2}$ and $\tilde{\sigma}_{1} \equiv \sigma_{1}-\sigma_{12}$. Therefore we see that the difference between calculations in the two currencies amounts to an overall shift in the volatilities by $\sigma_{12}$. But we have already seen that solutions of the PDE, Eq. (2.15), are invariant under such a translation. So we obtain a unique price function.

\subsection{Heath-Jarrow-Morton}

Let us consider the Heath-Jarrow-Morton framework [9]. The common approach is to postulate some forward rate dynamics and from there derive the prices of discount-bonds and other interest-rate instruments. But it is well-known that this model can also be formulated in terms of discount-bond prices [5]. Since discountbonds are tradables, this approach fits directly into our pricing formalism. Assume the following price process for the bonds ${ }^{i}$

$$
\frac{d_{t} P(t, T)}{P(t, T)}=\sigma(t, T, P) \cdot d W(t)+\alpha(t, T, P) d t .
$$

The drift and volatility should be homogeneous functions of degree zero in the bond prices in order to have a well-defined model, as was mentioned before. So they can only be functions of ratios of prices of bonds with different maturities. These prices should all be taken at time $t$, thereby excluding non-Markovian models. (NonMarkovian models can be incorporated into our formalism at the cost of more complexity. We will come back to this point in future work). Recall that the precise form of the drift-terms is not of any importance in deriving the claim price.

Now let us consider two examples of pricing in the HJM-model. For the sake of simplicity, we restrict our attention to Gaussian HJM models. In this case we have a bond satisfying

$$
\frac{d_{t} P(t, T)}{P(t, T)}=\sigma(t, T) \cdot d W(t)+\alpha(t, T) d t .
$$

So the drift and volatility only depend on $t$ and $T$. Both the (extended) Vasicek and the Ho-Lee model can be written in this form. In the case of the Vasicek model, the volatility function is given by

$$
\sigma(t, T) \equiv \frac{\sigma}{\alpha}\left(1-e^{-\alpha(T-t)}\right)
$$

when we take the cash bond (a.k.a. money market account) as numeraire [15]. Here $\alpha$ is the rate of mean reversion of the associated short rate. The Ho-Lee model is given by the limit $\alpha \rightarrow 0$. As a first example, we consider the price of an equity option with stochastic interest rates. We assume that the stock satisfies (again taking the cash bond as numeraire)

$$
\frac{d S}{S}=\sigma \cdot d W(t)+\alpha d t
$$

\footnotetext{
${ }^{\mathrm{i}}$ Here $d_{t}$ denotes the stochastic differential w.r.t. $t$.
} 
Now choosing $P(t, T)$ as a numeraire, we find the following PDE for the price of a claim (cf. Eq. (2.38))

$$
\left(\partial_{t}+\frac{1}{2}|\sigma-\sigma(t, T)|^{2} S^{2} \partial_{S}^{2}\right) V=0
$$

where $|v|$ denotes the length of the $k$-dimensional vector $v$. Using the techniques of Sec. 3.1, this leads to the following price for a call option with maturity $T$ and strike $K$

$$
V(P, S, t)=S \Phi\left(d_{1}\right)-K P \Phi\left(d_{2}\right)
$$

with

$$
d_{1,2}=\frac{\log \frac{S}{K P}}{\sqrt{\Sigma(t, T)}} \pm \frac{1}{2} \sqrt{\Sigma(t, T)}, \quad \Sigma(t, T)=\int_{t}^{T}|\sigma-\sigma(u, T)|^{2} d u .
$$

Remember that both $\sigma$ and $\sigma(t, T)$ are understood to be vectors.

As a second example, we consider the pricing of a European option on a linear combination of discount bonds, with a payoff defined by

$$
\mathrm{V}(T)=\left(\sum_{i} \beta_{i} P\left(T, T_{i}\right)\right)^{+}
$$

where the $\beta_{i}$ are constants. This example includes caplets, floorlets as well as swaptions. We will focus on the case of the extended Vasicek model. To calculate the value of this option at time $t$, we will use the bond with maturity $T$ as numeraire. The variance-covariance matrix then becomes

$$
\begin{aligned}
\Sigma_{i j} & =\int_{t}^{T}\left(\sigma\left(u, T_{i}\right)-\sigma(u, T)\right)\left(\sigma\left(u, T_{j}\right)-\sigma(u, T)\right) d u \\
& =\frac{\sigma^{2}}{2 \alpha^{3}}\left(1-e^{-\alpha\left(T_{i}-T\right)}\right)\left(1-e^{-\alpha\left(T_{j}-T\right)}\right)\left(1-e^{-2 \alpha(T-t)}\right) .
\end{aligned}
$$

The interesting property of the Vasicek model is that the matrix factorises: the singular decomposition is one-dimensional

$$
\Sigma_{i j}=\theta_{i} \theta_{j}, \quad \theta_{i}=\frac{\sigma}{\alpha \sqrt{2 \alpha}}\left(1-e^{-\alpha\left(T_{i}-T\right)}\right) \sqrt{1-e^{-2 \alpha(T-t)}} .
$$

So the option value becomes a one-dimensional integral

$$
\mathrm{V}(t)=\int\left(\sum_{i} P\left(t, T_{i}\right) \phi\left(z-\theta_{i}\right)\right)^{+} d z
$$

This integral can be evaluated by first identifying the regions of $z$ in which the argument is positive. Next, the integrals over these regions can be evaluated analytically.

Let us remark that in our model it is not necessary to use discount-bonds as fundamental tradables to model the interest rate market. One could equally well use other tradables such as coupon-bonds or swaps, being linear combinations of discount-bonds, or even caplets and swaptions. In our view, it seems to be less 
natural to model the LIBOR-rate directly, since this is not a traded object. In fact, $\delta$-LIBOR-rates are dimensionless quantities, defined as a quotients of discount bonds

$$
L(t, T)=\frac{P(t, T)-P(t, T+\delta)}{\delta P(t, T+\delta)} .
$$

In this respect, the name "LIBOR market-model" [12] seems a contradiction in terms.

\subsection{A trigger swap}

Let us now consider a somewhat more complicated example, a trigger swap [12]. Using again the results of Sec. 3.1, it becomes a trivial exercise to compute a price for this contract. This contract depends on four tradables $S_{i}$, and it is defined by its payoff function at maturity $T$

$$
f(S)=\left(S_{3}(T)-S_{4}(T)\right) \mathbf{1}_{S_{1}(T)>S_{2}(T)} .
$$

Here $\mathbf{1}_{A}$ is the characteristic function, which is unity if $A$ is true and zero otherwise. Note that both exchange options and binary options are special cases of this trigger swap. The former is found by setting $S_{3}=S_{1}$ and $S_{4}=S_{2}$, the latter by setting $S_{3}=P(t, T)$ and $S_{4}=0$. Let us assume that the $S_{i}$ satisfies

$$
\frac{d S_{i}}{S_{i}}=\sigma_{i}(t) \cdot d W(t)+\alpha_{i}(t) d t
$$

4 For this log-normal model, we can immediately write down the following formula for the price of the claim:

$$
V=\int_{S_{1} \phi\left(z-\theta_{1}\right)>S_{2} \phi\left(z-\theta_{2}\right)}\left(S_{3} \phi\left(z-\theta_{3}\right)-S_{4} \phi\left(z-\theta_{4}\right)\right) d z
$$

Here, the $\theta_{i}$ are given by a Cholesky decomposition of the integrated covariance matrix

$$
\Sigma_{i j}=\int_{t}^{T} \sigma_{i}(u) \cdot \sigma_{j}(u) d u=\theta_{i} \cdot \theta_{j} .
$$

We will omit the details of the evaluation of this integral. It is a straightforward application of the procedure described in Sec. 3.1. The result can be written as

$$
V\left(S_{1}, S_{2}, S_{3}, S_{4}, t\right)=S_{3} \Phi\left(d_{3}\right)-S_{4} \Phi\left(d_{4}\right),
$$

where

$$
d_{i}=\frac{\log \frac{S_{1}}{S_{2}}+\frac{1}{2}\left(\Sigma_{22}(t, T)-\Sigma_{11}(t, T)\right)+\Sigma_{1 i}(t, T)-\Sigma_{2 i}(t, T)}{\sqrt{\Sigma_{11}(t, T)-2 \Sigma_{12}(t, T)+\Sigma_{22}(t, T)}} .
$$

The reader can check that this result is again independent under gaugetransformations $\sigma_{i} \rightarrow \sigma_{i}-\lambda$, as it should be. Note that $V_{S_{1}}$ and $V_{S_{2}}$ are not in general equal to zero. This means that one needs a portfolio consisting of all four 
underlyings to hedge this claim. Now let us consider the special case of an exchange option, setting $S_{3}=S_{1}$ and $S_{4}=S_{2}$. In this case, the formulae reduce to

$$
V\left(S_{1}, S_{2}, t\right)=S_{1} \Phi\left(d_{1}\right)-S_{2} \Phi\left(d_{2}\right),
$$

where

$$
d_{1,2}=\frac{\log \frac{S_{1}}{S_{2}} \pm \frac{1}{2}\left(\Sigma_{11}(t, T)-2 \Sigma_{12}(t, T)+\Sigma_{22}(t, T)\right)}{\sqrt{\Sigma_{11}(t, T)-2 \Sigma_{12}(t, T)+\Sigma_{22}(t, T)}} .
$$

In Ref. [13] it is claimed that the value of an option to exchange two stocks has a dependence on the interest-rate term structure, or in other words, a dependence on bond-prices. It should be clear from the discussion above that this is in fact impossible, because neither the payoff nor the volatility functions make any reference to bonds. Therefore, the price of such an exchange option can be calculated in a market where bonds do not even exist.

\section{Conclusions and Outlook}

In the preceding sections we have clearly shown the advantages of a model formulated in terms of tradables only. In this formulation, the relativity of prices manifests itself as a homogeneity condition on the price of any contingent claim, and this fact can be exploited to bypass the usual martingale construction for the replicating trading-strategy. The result is a transparent general framework for the pricing of derivatives.

In this article we have restricted our attention to the problem of pricing European path-independent claims. The generalization to path-dependent and American options is straightforward. The former are discussed in more detail in Ref. [10]

Obviously, the applicability of the scale invariance is not restricted to models with Brownian driving factors. Currently we are considering alternative driving factors such as Poisson and Levy processes. We are also looking at implications for modelling incomplete markets. Finally the local scale invariance should also hold in markets with friction. This may serve as an extra guidance in the modeling of transaction-costs and restrictions on short-selling.

\section{Acknowledgment}

This work was carried out under the CWI-project MAS3.1 "Mathematical finance". The research of C.D.D. Neumann was partially supported by the SWON-program "Financial derivatives".

\section{Appendix A.}

We use stochastic differential equations to model the dynamics of the prices $x_{\mu}(t)$ of tradables. The governing equation is given by

$$
d_{t} x_{\mu}(t)=\sigma_{\mu}(x, t) x_{\mu}(t) \cdot d W(t)+\alpha_{\mu}(x, t) x_{\mu}(t) d t,
$$


with initial conditions $x_{\mu}(t)$ and $d W(t)$ denote $k$-dimensional Brownian motion with respect to some measure. The drifts $\alpha_{\mu}(x, t)$ and volatilities $\sigma_{\mu}(x, t)$ are assumed to be adapted to $x$ and predictable. For this equation to have a unique solution, we have to require some regularity conditions on the drift $\alpha_{\mu}(x, t)$ and volatility $\sigma_{\mu}(x, t)$. These can be stated as follows $[1,3,6]$ :

- Lipschitz condition: there exists a $K>0$ such that for all $x, y$ and $s \in[t, T]$

$$
\left|\alpha_{\mu}(x, s)-\alpha_{\mu}(y, s)\right|+\left|\sigma_{\mu}(x, s)-\sigma_{\mu}(y, s)\right| \leq K|x-y| .
$$

- Growth condition: there exists a $K$ such that for all $s \in[t, T]$

$$
\left|\alpha_{\mu}(x, s)\right|^{2}+\left|\sigma_{\mu}(x, t)\right|^{2} \leq K^{2}\left(1+|x|^{2}\right) .
$$

Although the Lipschitz condition above is global, it can in fact be weakened to a local version. If the growth condition is not satisfied, the solution may still exist up to some time $t^{\prime}$, where the solution $x_{\mu}(t)$ has a singularity and thus "explodes".

\section{References}

[1] L. Arnold, Stochastic Differential Equations, Wiley (1974).

[2] F. Black and M. Scholes, The pricing of options and corporate liabilities, Journal of Political Economy 81 (1973) 637-659.

[3] A. N. Borodin and P. Salminen, Handbook of Brownian Motion - Facts and Formulae, Birkhäuser (1996).

[4] P. Carr, Deriving derivatives of derivative securities, Working paper (1993).

[5] A. P. Carverhill, A simplified exposition of the Heath, Jarrow, and Morton model, Stochastics Reports 53 (1995) 227-240.

[6] C. W. Gardiner, Handbook of stochastic methods for physics, chemistry, and the natural sciences, Springer, 2nd edition (1985).

[7] H. Geman, N. El Karoui, and J.-C. Rochet, Changes of numeraire, changes of probability measure and option pricing, Journal of Applied Probability 32 (1995) 443-458.

[8] J. M. Harrison and S. R. Pliska, Martingales, stochastic integrals and continuous trading, Stochastic processes and their applications 11 (1981) 215-260.

[9] D. Heath, R. Jarrow, and A. Morton, Bond pricing and the term structure of interest: a new methodology for contingent claim valuation, Econometrica 60 (1992) 77-105.

[10] J. K. Hoogland and C. D. D. Neumann, Local scale invariance and contingent claim pricing II: Path-dependent contingent claims, IJTAF 4(1) (2001) 23-44.

[11] J. K. Hoogland and C. D. D. Neumann, Asians and cash dividends: Exploiting symmetries in pricing theory, CWI Technical Report MAS-R0019 (2000).

[12] F. Jamshidian, Libor and swap market-models and measures, Finance \& Stochastics 1 (1997) 293-330.

[13] C. Liu and D.-F. Wang, Exchange options and spread options with stochastic interest rates, http://xxx.lanl.gov/cond-mat/9904110 (1990).

[14] R. C. Merton, Theory of rational pricing, Bell Journal of Economics and Management Science 4 (1973) 141-183.

[15] M. Musiela and M. Rutkowski, Martingale methods in financial modelling, Springer (1997). 